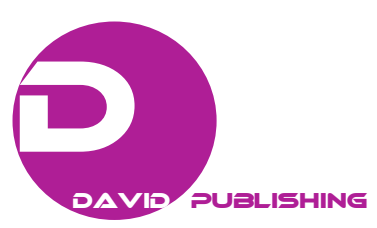

\title{
Knowledge State of Kisangani Avifauna (DRC)
}

\author{
Bapeamoni, A. and Upoki, A. \\ Département d'Ecologie et de Gestion des Ressources Animal (EGRA), Faculté des Sciences, Université de Kisangani (République \\ Démocratique du Congo), Congo
}

\begin{abstract}
Kisangani $\left(00^{\circ} 31^{\prime} \mathrm{N} 25^{\circ} 11^{\prime} \mathrm{E}\right.$; Alt. $\left.400 \mathrm{~m}\right)$ covers $1.910 \mathrm{~km}^{2}$. The primary habitat in the region is lowland rainforest. Ornithological studies in the region began with the American Museum of Natural History expedition in the early 1900s. The preliminary surveys showed an interesting wealth of iconic or endemic species (e.g. Congo Peacock, Afropavo congensis and Congo Sunbird, Nectarinia congoensis). The diversity of birds highligthed in these early surveys prompted the Faculty of Science at the University of Kisangani to begin to conduct systematic faunal studies of birds. Forest reserves, islands and disturbed environments were surveyed for birds. Capture and release and opportunistic observations were the primary survey methods. The present work provides a list of species encountered during surveys conducted at 5 sites from 1976-2014. A total of 267 species were recorded. These results provide a starting point to improve the state of knowledge about birds of Kisangani. However several natural forest sites were not surveyed: Uma, Banalia, Wania Rukula, Yangambi, Osiyo. The combination of several methods, sampling techniques and studies based on feathers, genetic material may be relevant to the assessment of avian biodiversity in this region.
\end{abstract}

Key words: State, knowledge, biodiversity, kisangani.

\section{Introduction}

From the scientific point of view, the ornithological exploration of the Kisangani area (Figure 1) began with an expedition by the American Museum of Natural History between 1909 and 1913; much of the material was collected in this region.

Although ranked among the "impenetrable" ecosystems by the first foreign researchers who conducted scientific research in Congo, these ecosystems have not hesitated to show that its birdlife is also interesting, among others, in symbolic and/or endemic species; for example Congo Peacock (Afropavo congensis) and Congo Sunbird (Cinnyris congoensis). Avifauna remained little known in dense lowland forests part of the Congolese central basin.

Since 1976, the Ecology and Animal Resources Management Laboratory (Laboratoire d'Ecologie et de Gestion des Ressources Animales) of the Science Faculty of Kisangani University began to conduct studies on the main zoological groups (mammals,

Corresponding author: Bapeamoni, A., research field: ecology. birds, invertebrates, insects, fishes, reptiles and amphibians). Bird studies have been conducted in several sites, mainly in Kisangani urban city (ecologically disturbed areas \& gardens), forest reserves (Masako \& Yoko) and island environments (Kungulu \& Mbiye).

With an area ranging between 1,910 and $2,109 \mathrm{~km}^{2}$, Kisangani (ck) $\left(0^{\circ} 31^{\prime} \mathrm{N} \quad 25^{\circ} 11^{\prime} \mathrm{E} ; 376-450 \mathrm{~m}\right)$ is located in the northeastern part of the Congolese central basin [1]. Apart from the extensive swamp, seats craft practices of rice-fish, the water system is dominated by the Congo, Tshopo and Lindi Rivers [2] These rivers have particular of having the islands and waterfalls in this region and the city extends on both banks of Congo River.

Five sites were been surveyed; two of these are protected areas north and south of the Congo River (Masako and Yoko, respectively). We also surveyed two large river islands.

Masako and Yoko are under the jurisdiction of Environment, Conservation of Nature, Water and Forests Ministry [3]. The protected areas are available for scientific surveys by Science of Kisangani 


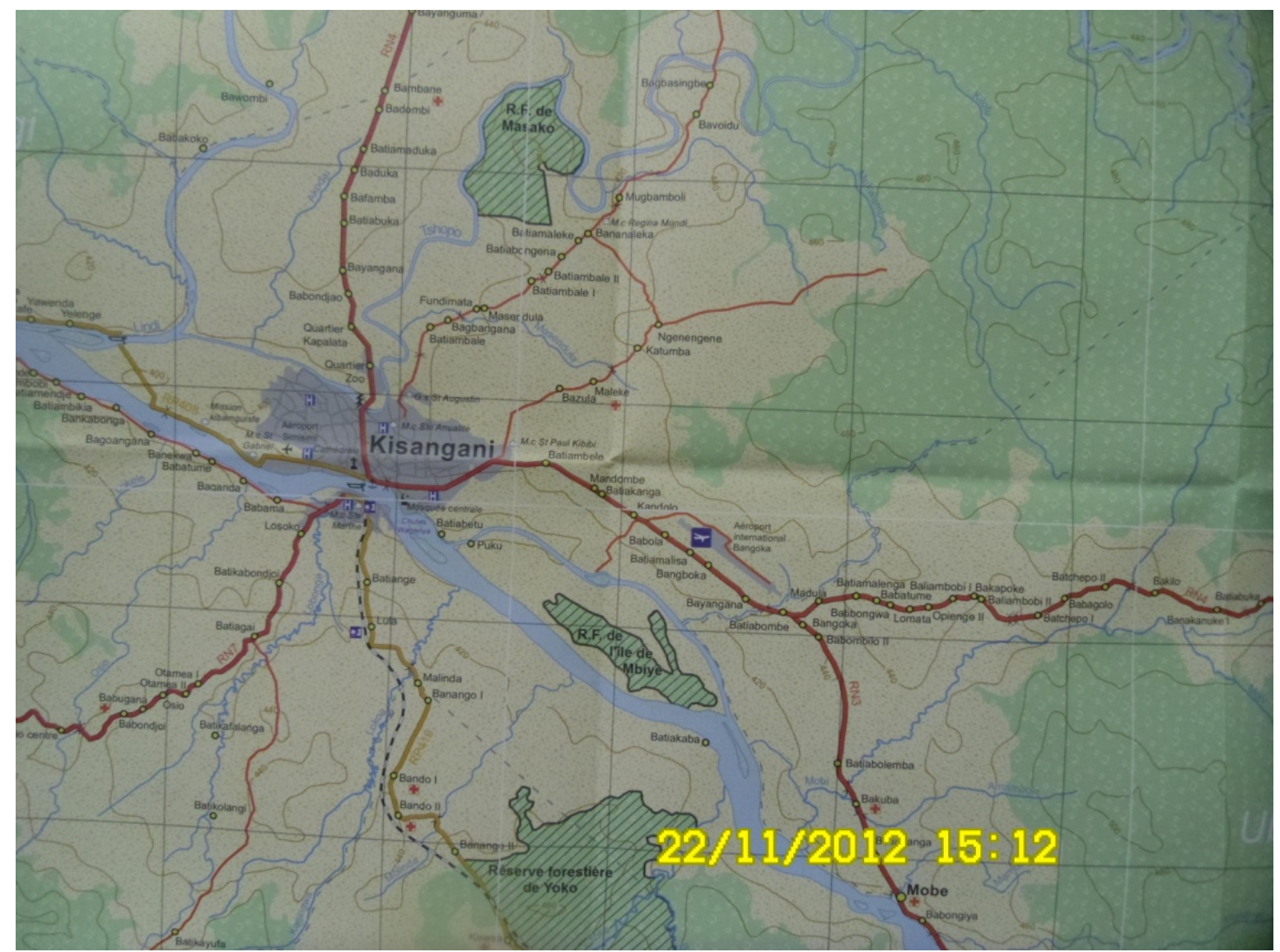

Fig. 1 Localization of Kisangani region.

University conducted studies on operation and sustainable management of forest ecosystems [4].

\section{Site Description}

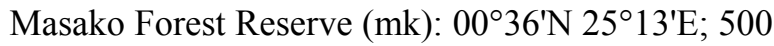
m; 2,105 ha; located in the large loop of Tshopo River at kilometer points fourteen (14) on the old Kisangani-Buta road.

Yoko Forest Reserve (yk): $00^{\circ} 17^{\prime} \mathrm{N} 25^{\circ} 17^{\prime} \mathrm{E} ; 400 \mathrm{~m}$; 6,975 ha; located on the left bank of the Congo River between kilometer points Twenty three (23) and Thirty two (32) on Kisangani-Ubundu road.

Kungulu (Kongolo) island (ik): $00^{\circ} 37^{\prime} \mathrm{N} 25^{\circ} 11^{\prime} \mathrm{E}$; $395 \mathrm{~m}$ upstream and $390 \mathrm{~m}$ downstream; located northwest of the Kisangani city to $15 \mathrm{Km}$ on Kisangani-Yangambi road at the merger of Lindi and Tshopo rivers in Congo River mouth. It has a length and a width respectively 4 and $0.6 \mathrm{~km}$.
Mbiye Island (im): $00^{\circ} 28^{\prime} \mathrm{N} 25^{\circ} 17^{\prime} \mathrm{E}$; $374 \mathrm{~m}$; 2,800 ha; located in south-east of Kisangani city. Its downstream edge is at the height of $4 \mathrm{~km}$ on Kisangani-Ituri road and its upstream edge is at the height of $22 \mathrm{~km}$ on Kisangani-Lubutu road.

The habitats in the Kisangani region are originally lowland rainforest. However, urbanization and traditional human activities such as shifting cultivation on slash and burn, logging gradually transforming the departure of landscape in the region. Overall, the region has a humid tropical climate like "Afi" of Koppen classification [5].

Bird's diversity known in Kisangani since 1976; their distribution according to the exploited sites; and sampling methods used are presents in this article.

\section{Results and Discussion}

A total of 267 species (Table 1 of Annex) have been 


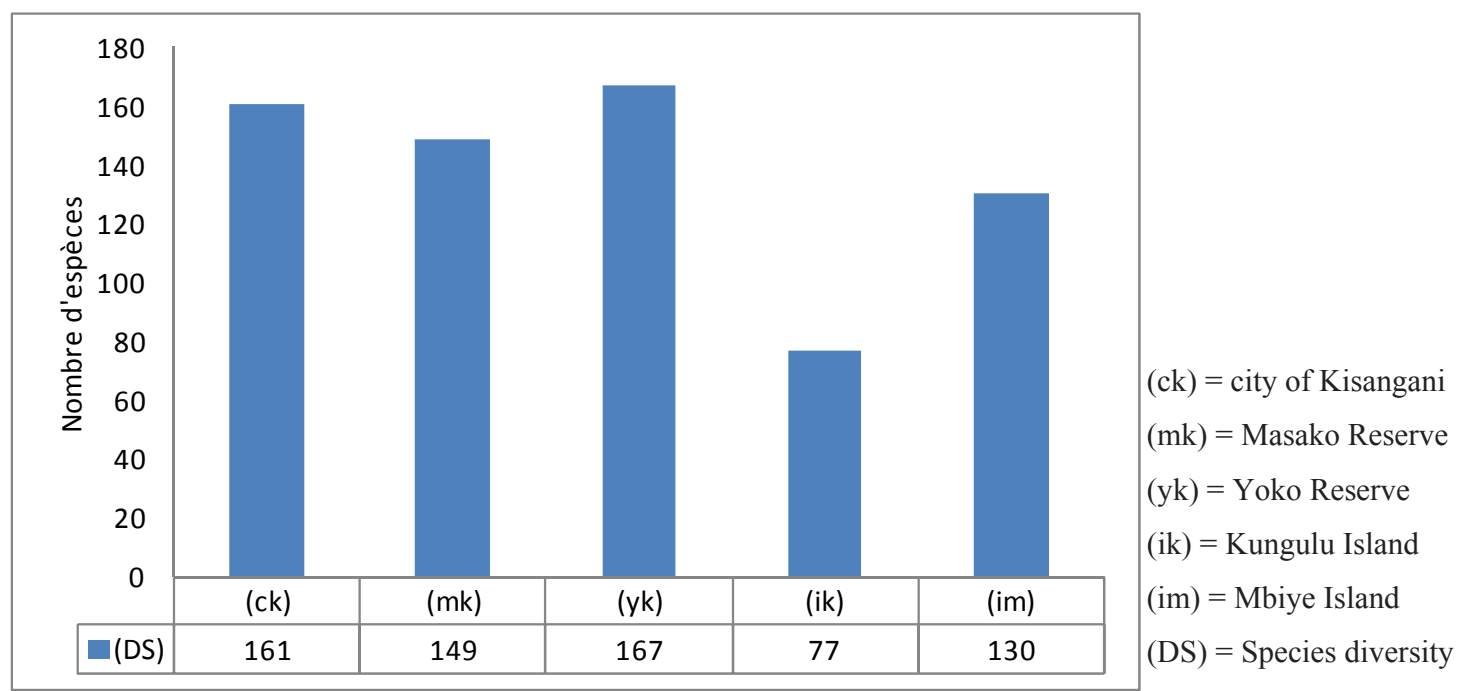

Fig. 2 Bird distribution across sites.

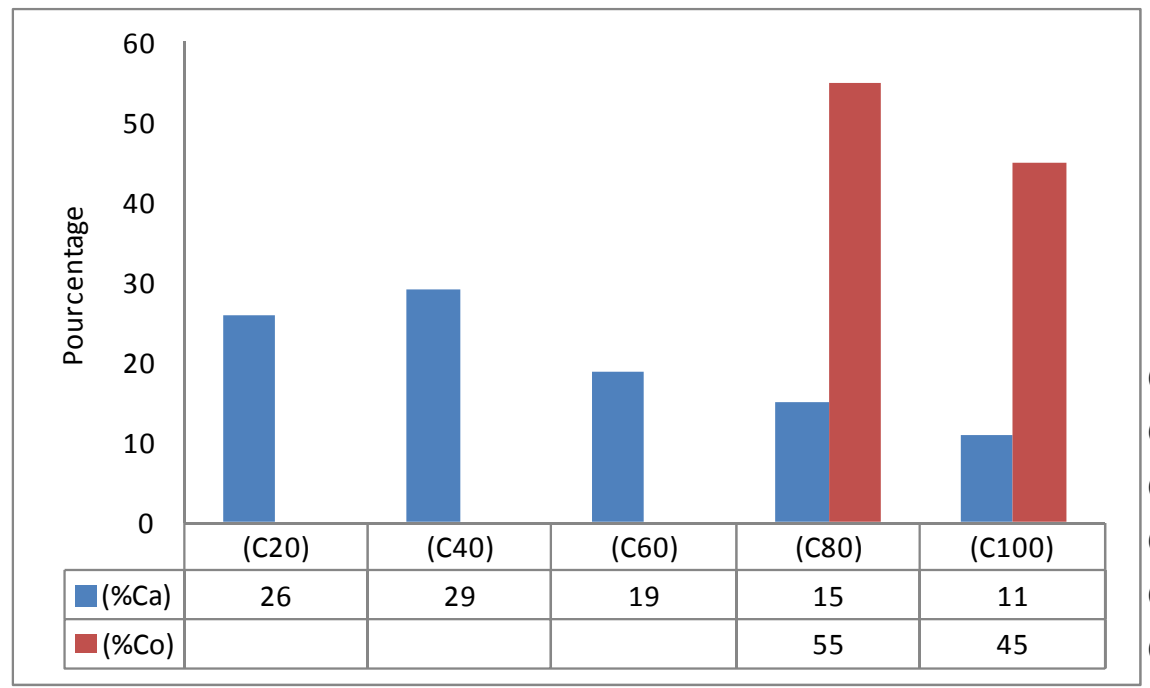

$(\mathrm{C} 20)=$ Constant at $20 \%$

$(\mathrm{C} 40)=$ Constant at $40 \%$

$(\mathrm{C} 60)=$ Constant at $60 \%$

$(\mathrm{C} 80)=$ Constant at $80 \%$

$(\mathrm{C} 100)=$ Constant at $100 \%$

$(\% \mathrm{Ca})=\%$ species stances by category

Fig. 3 Bird distribution of stances by category.

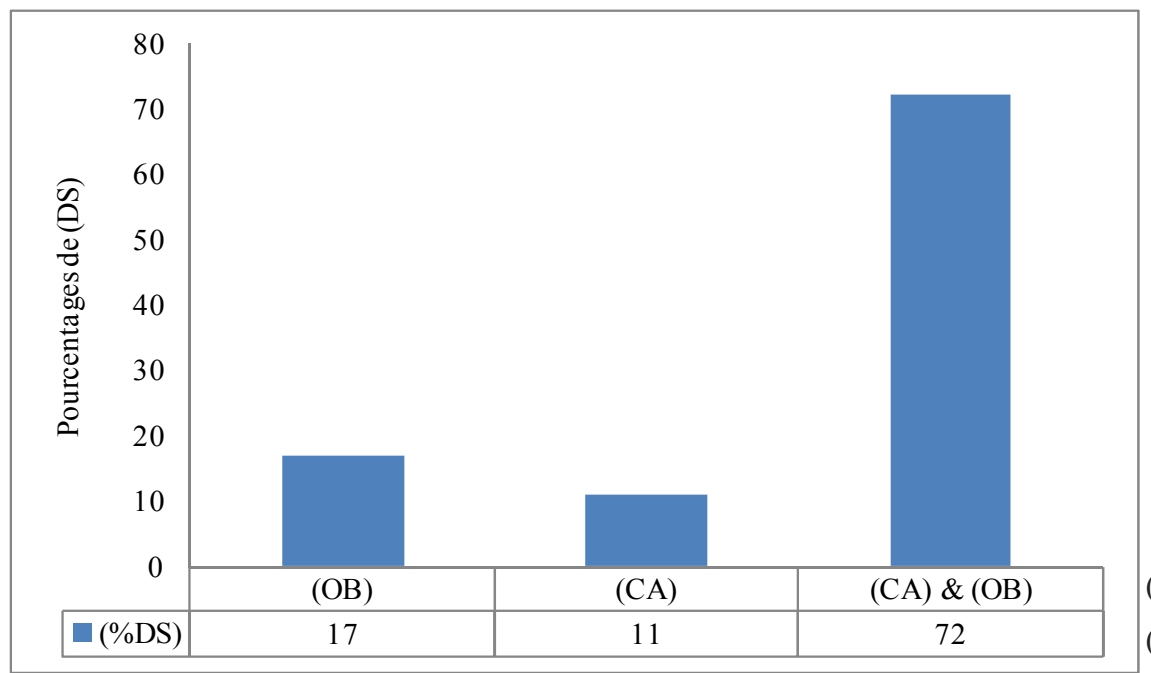

$(\mathrm{OB})=$ city of Kisangani

$(\mathrm{CA})=$ Reserve Forest Masako

Fig. 4 Bird distribution methods Sample Rate. 
recorded in Kisangani. The distribution of bird wealth and the methods used are shown by the following figures.

Avian richness achieved so far in Kisangani area puts in evidence a growing interest in studying the avifauna of this Congo Basin's part. In this region, Bashige and Dobonnet [6] estimated 300 species excluding water birds and migrants. Currently, Kisangani presented $39 \%$ of Katanga province's avifauna [7], 24\% of the whole DRC [8]. This study focused on lowland rainforest and forest edge habitats. By incorporating other habitats, we are certain that we will uncover higher estimates of bird diversity in the region. Yoko (yk), urban city (ck) and masako (mk) are high species diversity sites. They are followed by Mbiye (im) and Kunguklu (ik) Islands.

Environmental disturbances (urbanization, agricultural activities in Kisangani tend to restrict forest dependent birds residents at Yoko and Masako, while Kisangani city becomes a preferential site for ruderal and migratory species [9]. The Mbiye Island has a high species bird number relative to Kungulu. The island biodiversity is closely related to the area and the environmental condition [10].

\section{Conclusions}

In Kisangani avian studies are still at the beginning. The first results show the value of understanding the distribution of avian diversity in this part of the Congo Basin; a region that continues to be the subject to ecological disturbance jeopardizing the future of biodiversity.

\section{References}

[1] Ndjele, M.-B. 1988. Les éléments phytogéographiques endémiques de la forêt vasculaire du Zaïre, Thèse dde doctorat, Université Libre Bruxelles, Bruxelles, 528.

[2] Nyakabwa, M. 1982. Phytocénose de l'écosystème urbain de Kisangani, Thèse de doctorat, 1ère partie, Université de Kisangani, Kisangani, 10-23.

[3] Juakaly, M. 2007. Résilience et écologie des Araignées du sol d'une forêt équatoriale de Basse altitude (Réserve forestière de Masako, Kis., R.D.C), Thèse de doctorat Université de Kisangani, Kisangani, 18-26.

[4] Boyemba, B., 2011. Ecologie de Pericopsis elata (Harms) van Meeuweb (Fabaceae) arbre de forêt tropicale à distribution agrégée, Thèse de doctorat Université Libre de Bruxelles, Bruxelles, 181.

[5] Nshimba, S. M., Bamba, I., Iyongo, W., Ndjele, M. B., and Bogaert, J. 2011. Analysis of the degradation of the forest ecosystem of Mbiye Island (Kisangani, RDC), Kisangani, 100-10.

[6] Bashige, E., et Debonnet, G. 2004. La Biodiversité en RDC. P31-64, in MRAC (réd) compte rendu de l'Exposition sur le Congo, Nature en RDC, Tervuren, 23 novembre 2004 au 2 octobre 2005, Service de publication de Musée Royal de l'Afrique Centrale, Tervuren, 160.

[7] Louette, M., et Hasson, M. 2011. "Les oiseaux du Katanga." Studies in Afrotropical zoology 296: 404.

[8] Bapeamoni, A. 2014. Biodivesrité et densité des nids des Oiseaux dans un dispositif permenant dans Yoko (Ubundu, RDC), Thèse de doctorat, Université de Kisangani, Kisangani, 113.

[9] Bapeamoni, A. 2008. Activités hivernales du Guêpier à gorge blanche (Merops albicollis, Meropidae), DEA, Université de Kisangani, Kisangani, 56.

[10] Mac Athur, R and Levins, R. 1967. The limiting similiarity convergence and divergence of coexisting species, American Naturalist, 101: 377-85. 


\begin{tabular}{|c|c|c|c|}
\hline $\mathrm{N}^{\circ}$ & & Scientific names & \\
\hline & Accipiter castanilius & 48 Bycanistes cylindricus* & 95 Cyanomitra olivacea \\
\hline & Bycanystes sharpei & 49 Bycanistes subcylindricus* & 96 Cypsiurus parvus \\
\hline & Accipiter erythropus & 50 Camaroptera brevicaudata & 97 Deleornis axillaris \\
\hline & Accipiter melanoleucus & 51 Camaroptera chloronata & 98 Delichon urbicum \\
\hline & Accipiter minullus & 52 Campephaga flava & 99 Dendropicos fulscenscens \\
\hline 6 & Accipiter tachiro & 53 Campephaga phoenicea & 100 Dendropicos gabonensis \\
\hline 7 & Accipiter tousseneli & 54 Campethera nivosa & 101 Dicrurus adsimilis \\
\hline 8 & Acrocephalus arundinaceus & 55 Caprimulgus batesi & 102 Dicrurus atripennis \\
\hline 9 & A crocephalus rufescens & 56 Caprimulgus fossii & 103 Dicrurus coracinus \\
\hline 10 & Acrocephalus scirpyapaenus & 57 Caprimulgus inomatus & 104 Dryotriochis spectabilis \\
\hline 11 & Acrocephalus shoenobaenus & 58 Centropus grillii & 105 Dyaphorophya castanea \\
\hline 12 & Actophilornis africana & 59 Centropus senegalensis & 106 Dyaphorophya tonsa \\
\hline 13 & Alcedo cristata & 60 Ceraptogimna atrata & 107 Egretta alba \\
\hline 14 & Alcedo leucogaster & 61 Ceryle rudis & 108 Egretta garzeta \\
\hline 15 & Alcedo quadribrachys & 62 Ceuthmochares aereus & 109 Elanus caeroleus \\
\hline 16 & Alethe castanea & 63 Ceyx lecontei & 110 Estrilda melpoda \\
\hline 17 & Alethe poliocephata & 64 Ceyx pictus & 111 Estrilda nonnula \\
\hline 18 & Alethe poliophrys & 65 Chalcomitra rubescens & 112 Euplectes afer \\
\hline 19 & Amblyospiza albifrons & 66 Charadrius hiaticula & 113 Euplectes hordeaceus \\
\hline 20 & Amauromis flavirostra & 67 Charadrius forbesi & 114 Eurystomus glaucurus \\
\hline 21 & Anhinga rufa & 68 Charadrius marginatus & 115 Eurystomus gularis \\
\hline 22 & Anastomus lamelligerus & 69 Chlorocichla flavicollis & 116 Falco biarmicus \\
\hline 23 & Andropadus gracilirostris & 70 Chlorocichla simplex & 117 Falco subbuteo \\
\hline 24 & Andropadus gracilis & 71 Chrysococcyx klaas & 118 Falco tinnunculus \\
\hline 25 & Andropadus latirostris & 72 Chrysococcyx caprius & 119 Gallinago gallinago \\
\hline 26 & Andropadus virens & 73 Chrysococcyx cupreus & 120 Gallinula angulata \\
\hline 27 & Andropardus curvirostris & 74 Cinnyris chloropygius & 121 Glareola nuchalis \\
\hline 28 & Anthreptes collaris & 75 Cinnyris batesi & 122 Glaucidium sjostedi \\
\hline 29 & Anthreptes rectirostris & 76 Cinnyris minulla & 123 Guttera pucherani \\
\hline 30 & Apaloderma narina & 77 Cinnyris superba & 124 Gymnobuco bonapartei \\
\hline 31 & Apus apus & 78 Circus acrugenesus & 125 Gymnobuco sladeni \\
\hline 32 & Apus affinis & 79 Cisticola anonymus & 126 Gypohierax angolensins \\
\hline 33 & Ardea cinerea & 80 Cisticola brachyptera & 127 Halcyon badia \\
\hline 34 & Ardea goliath & 81 Cisticola marginatus & 128 Halcyon malimbicus \\
\hline 35 & Ardea melanocephala & 82 Clamotor levaillantii & 129 Halcyon senegalensis \\
\hline 36 & Ardea purpurea & 83 Columba unicincta & 130 Hiliaetus vocifer \\
\hline 37 & Ardea sturmii & 84 Coracias caudata & 131 Hirundo abyssinica \\
\hline 38 & Ardeola ralloides & 85 Corvus palvus & 132 Hirundo angolensis \\
\hline 39 & Baeopogon indicator & 86 Corythacoela cristata* & 133 Hirundo nigrita \\
\hline 40 & Bias musucus & 87 Criniger barbatus & 134 Hirundo rustica \\
\hline 41 & Bleda eximia & 88 Criniger calurus & 135 Hirundo semirufa \\
\hline 42 & Bleda syndactylis & 89 Criniger ndussumensis & 136 Hirundo senegalensis \\
\hline 43 & Bleda ugandae & 90 Cucu canorus & 137 Hirundo smithii \\
\hline 44 & Brachycope anomala & 91 Cuculus clamosus & 138 Hylia presina \\
\hline 45 & Bubulcus ibis & 92 Cuculus salitarius & 139 Illadopsis albipectus \\
\hline & Buccanodon duchailluis & 93 Cyanomitra cyonoleama & 140 Illadopsis fulvescens \\
\hline 47. & Bycanistes albotibialis* & 94 Cyanomitra obscura & 141 Illadopsis puveli \\
\hline
\end{tabular}

\begin{tabular}{|c|c|c|c|c|}
\hline $\mathrm{N}^{\circ}$ & & & Scientific names & \\
\hline 142 & Illadopsis rufipennis & 184 & Neocossyphus rufus & 226 Pteronetta hartlaubi \\
\hline 143 & 3 Indicator exilis & 185 & Nicator chloris & 227 Pycnonotus balbatus \\
\hline 144 & Indicator maculatus & 186 & Nicator vireo & 228 Quelea cardinalis \\
\hline 145 & Indicator minor & 187 & Nigrita bicolor & 229 Quelea erythrops \\
\hline 146 & Ispidina picta & 188 & Nigrita canicapillus & 230 Riparia cincta \\
\hline 147 & Ixonotus guttatus & 189 & Nigrita fusconotus & 231 Riparia paludicola \\
\hline 148 & Kaupifalco monogrammicus & 190 & Nigrita luteifrons & 232 Riparia riparia \\
\hline 149 & Lamprotornis splendidus & 191 & Oriolus brachyrhynchus & 233 Sarkidiornis melanotos \\
\hline 150 & Laniarius leucorhynchus & 192 & Oriolus nigripennis & 234 Sartothrura pulchra \\
\hline 151 & Laniarus splendilus & 193 & Oriolus oriolus & 235 Sasia africana \\
\hline 152 & Lanius collaris & 194 & Parmoptila rubrifrons & 236 Smithornis capensis \\
\hline 153 & Bubo poensis & 195 & Passer griseus & 237 Spermophaga heamatina \\
\hline 154 & Lonchura bicolor & 196 & Phalacrocorax africanus & 238 Spermophaga poliogenus \\
\hline 155 & Lonchura cucullata & 197 & Phyllastrephus albigularis & 239 Stiphornis erythrothorax \\
\hline 156 & Lonchura frigilloïdes & 198 & Phyllastrephus baumanni & 240 Stiphrornis sangensis \\
\hline 157 & Macrodipteryx longipennis & 199 & Phyllastrephus icterinus & 241 Stiphrornis xanthogaster \\
\hline 158 & Macrodipteryx vexillarius & 200 & Phyllastrephus xavieri & 242 Stizorhina fraseri \\
\hline 159 & Malimbus malimbicus & 201 & Phyllolais pulchella & 243 Stocopelia peli \\
\hline 160 & Malimbus nictens & 202 & Phylloscopus trochilus & 244 Streptopelia semitorquata \\
\hline 161 & Faseria cinerascens & 203 & Pireneste ostrinus & 245 Strix woodfordii \\
\hline 162 & Fraseria ocreata & 204 & Pitta reichenowi & 246 Thalassornis leuconotus \\
\hline 163 & Megabyas flammulatus & 205 & Platysteria cyanea & 247 Terpsiphone batesi \\
\hline 164 & Megaceryle maximus & 206 & Platysteria laticineta & 248 Terpsiphone rufiventer \\
\hline 165 & Melaenornis pammelaina & 207 & Plectropterus gambensis & 249 Terpsiphone rufucinerea \\
\hline 166 & Melichneustes robustus & 208 & Ploceus cuculatus & 250 Terpsiphone viridis \\
\hline 167 & Merops albicollis & 209 & Ploceus nigerrimus & 251 Tockus fasciatus* \\
\hline 168 & Merops apiaster & 210 & Ploceus pelzeni & 252 Treron australis* \\
\hline 169 & Merops muelleri & 211 & Pogoniulus atroflavus & 253 Treron calva \\
\hline 170 & Merops persecus & 212 & Pogoniulus leucolamysta & 254 Tringa glareola \\
\hline 171 & Merops superciliosus & 213 & Pogoniulus scolopaceus & 255 Tringa nebularia \\
\hline 172 & Merops variegatus & 214 & Pogoniulus subsulphureus & 256 Tringa ochropus \\
\hline 173 & Milvus migrans & 215 & Poicephalus gulielmi & 257 Tringa stagnatilis \\
\hline 174 & Milvus milvus & 216 & Glareola pratincola & 258 Tringa pugnax \\
\hline 175 & Motacilla aguimp & 217 & Poliohierax semitorquatus & 259 Tringa hypoleucos \\
\hline 176 & Motacilla flava & 218 & Porphyrula alleni & 260 Trochocercus nitens \\
\hline 177 & Muscicapa cassini & 219 & Polyboroides tupus & 261 Tropicranus albocristatus \\
\hline 178 & Muscicapa comitata & 220 & Prinia leucopogon & 262 Turdus olivaceus \\
\hline 179 & Muscicapa striatta & 221 & Prinia subflava & 263 Turdus pelios \\
\hline 180 & Musophaga rossae & 222 & Psalidoprocne nictens & 264 Turtur afer \\
\hline 181 & Nattapus auritus & 223 & Psalidoprocne pristoptera & 265 Turtur brehmeri \\
\hline 182 & Neocossyphus fraseri & 224 & Pseudhirundo griseopyga & 266 Turtur tympanis tria \\
\hline 183 & Neocossyphus poensis & 225 & Psittacus erythacus* & 267 Vidua macroura \\
\hline
\end{tabular}

